\title{
Termoterapia e Quimioterapia de Banana 'Prata-Anã' Associadas à Temperatura de Armazenamento no Controle de Podridões em Pós-Colheita*
}

\author{
Wilson da S. Moraes ${ }^{1 * *}$, Laércio Zambolim², Juliana D. Lima ${ }^{3}$, Francisco Xavier R. do Vale ${ }^{2}$ \\ \& Luiz C. C. Salomão ${ }^{4}$ \\ ${ }^{1}$ Agência Paulista de Tecnologia dos Agronegócios, Laboratório Primário de Sanidade Animal e Vegetal de Registro, \\ UPD, APTA, Av. Wild José de Souza, 454, Centro, Registro, SP, CEP 11900-000, Fax: (013) 3821-2282, e-mail: \\ wilson_moraes@uol.com.br; ${ }^{2}$ Departamento de Fitopatologia, Universidade Federal de Viçosa; ${ }^{3}$ Unidade Diferenciada \\ de Registro, Universidade Estadual Paulista; ${ }^{4}$ Departamento de Fitotecnia, Universidade Federal de Viçosa
}

(Aceito para publicação em 24/05/2005)

Autor para correspondência: Wilson da Silva Moraes

MORAES, W.S., ZAMBOLIM, L., LIMA, J.D., VALE, F.X.R. \& SALOMÃO, L.C.C. Termoterapia e quimioterapia de banana 'Prata-Anã' associadas à temperatura de armazenamento no controle de podridões em pós-colheita. Fitopatologia Brasileira 31:017-022. 2006.

\begin{abstract}
RESUMO
O objetivo deste estudo foi avaliar o efeito da termoterapia $\left(56^{\circ} \mathrm{C}\right.$ por $6 \mathrm{~min}$ ) e quimioterapia (propiconazole $250 \mathrm{ml.}^{-1}$ ) associado com temperatura de armazenamento (temperatura ambiente, $18{ }^{\circ} \mathrm{C}$ e $13{ }^{\circ} \mathrm{C}$ ) no controle de podridões de bananas (Musa spp.) 'Prata-Anã' (AAB) em pós-colheita. Os tratamentos apresentaram diferenças significativas na percentagem de área lesionada por fruto, perda de peso e coloração externa da casca em todas as temperaturas de armazenamento. A quimioterapia e a combinação termoterapia e quimioterapia evitaram a manifestação de podridões nas três condições de armazenamento, enquanto a termoterapia reduziu a percentagem de área lesionada por fruto de $98 \%$ para $11 \%$ em temperatura ambiente, de $8 \%$ para $7 \%$ em $18{ }^{\circ} \mathrm{C}$ e de $10 \%$ para $0 \%$ em $13{ }^{\circ} \mathrm{C}$, sendo mais eficiente sob a temperatura de $13{ }^{\circ} \mathrm{C}$. Frutos não tratados perderam $25 \%, 10 \%$ e $3 \%$ de peso e atingiram a cor 7,5 e 1 em temperatura ambiente, $18{ }^{\circ} \mathrm{C}$ e $13{ }^{\circ} \mathrm{C}$, respectivamente. Frutos tratados com termoterapia e quimioterapia perderam 24, 11 e 5\% e 20, 10 e 3\%, e atingiram índice médio de cor 4 e 3,5, respectivamente. O período de conservação foi estendido para 18 , 24 e 45 dias em temperatura ambiente, $18^{\circ} \mathrm{C}$ e $13^{\circ} \mathrm{C}$, respectivamente. A combinação dos métodos evitou a manifestação de podridões, reduziu a perda de peso e manteve a cor da casca e a qualidade dos frutos.
\end{abstract}

Palavra-chave adicionais: tratamento térmico, tratamento químico, antracnose.

\begin{abstract}
Thermotherapy and chemotherapy of banana 'Prata-Anã' associated with storage temperature in post harvest rot control

The objective of this study was to evaluate the effects of thermotherapy $\left(56^{\circ} \mathrm{C}\right.$ for $6 \mathrm{~min}$ ) and chemotherapy (propiconazole $250 \mathrm{ml.}^{-1}$ ) associated with storage temperature (environmental temperature, $18{ }^{\circ} \mathrm{C}$ and $13^{\circ} \mathrm{C}$ ) in the rot control of postharvest banana (Musa spp.) fruits. Treatments have shown significant differences in the percentage of peel area with lesions per fruit, weight loss and external peel coloration in all storage temperatures. Chemotherapy and the combination of both thermotherapy and chemotherapy avoided rot manifestation in the three storage temperatures. Thermotherapy, after 15 days, reduced the percentage of peel area with lesions per fruit from $98 \%$ to $11 \%$ at environmental temperature, from $8 \%$ to $7 \%$ at $18{ }^{\circ} \mathrm{C}$ and $7 \%$ to $0 \%$ at $13{ }^{\circ} \mathrm{C}$, being most efficient at $13{ }^{\circ} \mathrm{C}$. Non-treated fruits lost $25 \%, 10 \%$ and $3 \%$ of their weight and got color 7,5 and 1 at environmental temperature, $18{ }^{\circ} \mathrm{C}$ and $13{ }^{\circ} \mathrm{C}$ respectively, after 15 day. Fruits treated with thermotherapy and chemotherapy lost 24, 11 and 5\% and 20, 10 and 3\% of weight and got color 4 and 3,5, respectively. The conservation period was extended to 18,24 and 45 days at environmental temperature, $18^{\circ} \mathrm{C}$ and $13{ }^{\circ} \mathrm{C}$, respectively. The combination of methods avoided rot manifestation, reduced weight loss and maintained external peel coloration and fruit quality.
\end{abstract}

Additional keywords: thermal treatment, chemical treatment, antracnose.

\section{INTRODUÇÃO}

A banana (Musa spp.) 'Prata-Anã' (AAB) vem ganhando importância com potencial para atender o mercado externo em decorrência dos ótimos atributos de qualidade.

\footnotetext{
*Parte da Tese de Doutorado do primeiro autor. Universidade Federal de Viçosa (1999)

**Bolsista do CNPq
}

Entretanto, o grande desafio está no curto período de conservação dos frutos dessa cultivar em pós-colheita, seguido da ação de microrganismos que aceleram sua deterioração (Alves et al, 1995).

A principal forma de controle das doenças em póscolheita é o uso de fungicidas que apresenta alguns inconvenientes, como a forma de aplicação (Wisniewski \& Wilson, 1992; Thompson, 1994), o surgimento de patógenos 
resistentes (Spalding \& Reeder, 1972) e as pressões sócioeconômicas e têm reduzido a oportunidade do controle baseado apenas no uso de fungicidas. Alternativas visando estratégias para não "queimar" o produto ou a diminuição do seu uso têm mostrado resultados promissores (Spalding \& Reeder, 1972; Johnson \& Sangchote, 1994).

A termoterapia ou tratamento térmico ou hidroterapia é um método alternativo que tem sido usado há vários anos para controle de doenças fúngicas em pós-colheita (Couey, 1989). Entretanto, as maiores limitações ao seu uso referemse à falta de proteção residual contra a recontaminação por patógenos oportunistas e às injúrias promovidas no hospedeiro.

A refrigeração é outro método alternativo para controle de podridões em pós-colheita, uma vez que baixas temperaturas retardam os processos fisiológicos, associados ao amadurecimento (Durigan, 1994; Labavitch, 1998) e o crescimento de fungos, permitindo estender o período de comercialização e melhorar a qualidade dos frutos (Jones \& Slabaugh, 1994). Seus benefícios foram comprovados em cultivares de banana tipo exportação, como nanica e nanicão (Jankovic, 1988; Hewage et al., 1994; Hochaus, 1994), sendo recomendado $12{ }^{\circ} \mathrm{C}$ a $15{ }^{\circ} \mathrm{C}$ para conservação de frutos de banana durante duas a quatro semanas (Johnson \& Sangchote, 1994; Kader, 1994).

A combinação de métodos de controle pode mostrar efeito aditivo ao aumentar a eficiência de controle das podridões e prolongar a vida pós-colheita dos frutos, como no caso da termoterapia, onde o controle é geralmente parcial, necessitando da suplementação de fungicidas durante ou após o tratamento para atingir nível de controle satisfatório (Jacobi et al., 1994). Fungicidas como benomil, thiabendazole e imazalil são freqüentemente adicionados à água quente para melhorar a eficiência de controle de doenças em pós-colheita (Spalding \& Reeder, 1972).

A termoterapia foi suficiente para controlar podridão peduncular em manga (Mangifera indica L.) e mamão (Carica papya L.) armazenados a $13{ }^{\circ} \mathrm{C}$, e quando associada ao tratamento com fungicida benomil ou procloraz foi efetiva contra podridões causadas por Colletotrichum musae em banana e Alternaria sp. em manga (Liu et al., 1997). Bananas tratadas com procloraz foram menos afetadas por podridões da coroa, quando comparadas com bananas armazenadas a $13^{\circ} \mathrm{C}$ durante duas semanas (Smith \& Anderson, 1975). Assim, vislumbra-se a utilização da quimioterapia e, ou termoterapia associada com temperaturas de armazenamento inferiores que não causem injúrias e prolonguem a vida dos frutos.

Pelo exposto, esse trabalho teve por objetivo avaliar a utilização da termoterapia e quimioterapia isolada ou combinada com temperatura de armazenamento visando o controle integrado de podridões e o aumento da vida póscolheita da banana 'Prata-Anã'.

\section{MATERIAL E MÉTODOS}

\section{Material Vegetal}

Cachos de banana 'Prata-Anã' em estádio pré- climatérico foram colhidos de plantações comerciais com dois anos de idade, no município de Jaíba, MG. Em seguida, despencados e as pencas selecionadas, acondicionadas em caixas plásticas, protegidas do sol com folhas de bananeiras e transportadas por caminhão aberto em 12 h, até o Laboratório de Pós-Colheita da Universidade Federal de Viçosa. Visando maior uniformidade durante a maturação, selecionaram-se as pencas centrais dos cachos, rejeitando-se as duas pencas proximais e as três pencas distais.

\section{Tratamentos utilizados}

a) Térmico - imersão de buquês (cinco frutos) em água quente a $56{ }^{\circ} \mathrm{C}$, durante 6 min e resfriamento em água à temperatura ambiente durante $10 \mathrm{~min}$;

b) Fungicida - imersão de buquês em suspensão do fungicida propiconazole a $250 \mathrm{ml.}^{-1}$ à temperatura ambiente, durante 3 min;

c) Térmico+Fungicida - imersão dos buquês em água quente a $56{ }^{\circ} \mathrm{C}$ durante 6 min, seguida da imersão em suspensão do fungicida propiconazole a $250 \mathrm{ml}^{-\mathrm{l}^{-1}}$ à temperatura ambiente durante $3 \mathrm{~min}$;

d) Controle - imersão dos buquês em água à temperatura ambiente.

Após os tratamentos, os buquês foram divididos em três lotes e armazenados sob condição ambiente de $23-30{ }^{\circ} \mathrm{C}$ (68 a $82 \%$ UR), a $18{ }^{\circ} \mathrm{C}\left(60\right.$ a $85 \%$ UR) e a $13{ }^{\circ} \mathrm{C}(80$ a $95 \%$ UR).

\section{Avaliação dos dados}

Os lotes contendo 64 buquês (16 buquês por tratamento) foram avaliados quanto à perda de peso, evolução da cor externa da casca, tempo necessário para manifestação de podridões e a severidade de podridões. O início de manifestação dos sintomas e a percentagem da área lesionada por fruto ou podridões (\% ALF), a cor dos buquês e a percentagem de perda de peso foram avaliadas a zero, três, seis, nove, 12 e 15 dias após os tratamentos. As avaliações continuaram até 24 e 45 dias para comparar os tratamentos armazenados a 18 e $13{ }^{\circ} \mathrm{C}$, respectivamente. A severidade das podridões foi determinada com auxílio de uma escala diagramática, desenvolvida e validada para este estudo, com variações de 0 a $64 \%$ da área lesionada por fruto. A evolução da cor dos buquês foi determinada com auxílio da escala visual descrita por Dadzie \& Orchard (1996), com índices variando de 1 a 7 .

\section{Delineamento Experimental e Análise Estatística}

O experimento foi disposto em esquema fatorial $4 \times 3$ (quatro tratamentos e três temperaturas de armazenamento) no delineamento inteiramente casualizado. Os dados de porcentagem de área lesionada / fruto, perda de peso e índice de cor foram transformados em raiz quadrada de $\mathrm{x}+1$ para a normalização de sua distribuição, porém nos gráficos foram apresentadas médias originais. Para comparação das médias utilizou-se o teste Tukey, ao nível de 5\% de probabilidade. 


\section{RESULTADOS E DISCUSSÃO}

A percentagem de área lesionada por fruto e de perda de peso foram afetadas pela termoterapia $\left(56{ }^{\circ} \mathrm{C}\right.$ por 6 min) e quimioterapia (propiconazole $250 \mathrm{ml}^{l^{-1}}$ por $3 \mathrm{~min}$ ), isoladas ou combinadas, e pelas temperaturas de armazenamento sob condições ambientes $\left(\mathrm{T}=23-30{ }^{\circ} \mathrm{C}\right.$ e UR $\left.=68-82 \%\right), 18{ }^{\circ} \mathrm{C}$ (UR $=60$ a $80 \%)$ e $13^{\circ} \mathrm{C}(\mathrm{UR}=80$ a $95 \%)$. Houve uma redução acentuada na percentagem da área lesionada em frutos tratados com a termoterapia e, ou quimioterapia e posteriormente armazenados à temperatura de $13^{\circ} \mathrm{C}$ (Figura 1$)$.

A termoterapia reduziu a área lesionada por fruto de $98 \%$ para $11 \%$ (ambiente), de $8 \%$ para $7 \%\left(18{ }^{\circ} \mathrm{C}\right)$ e de $10 \%$ para $0 \%\left(13{ }^{\circ} \mathrm{C}\right)$, em relação ao controle. O tratamento químico e o térmico+químico preveniram completamente as podridões nas condições de armazenamento testadas (Figura 1).

Os resultados indicam um aumento significativo na eficiência da termoterapia na medida em que houve redução da temperatura de armazenamento. Tanto que, apenas a 13 ${ }^{\circ} \mathrm{C}$, mostrou-se efetivo no controle completo das podridões, enquanto que sob condições ambientes e a $18^{\circ} \mathrm{C}$ apresentou apenas controle parcial, porém com redução significativa da área lesionada por fruto. Contudo, mostrou-se suficiente para substituir o tratamento químico, com vistas a atender mercados mais exigentes quanto à presença de resíduos de agrotóxicos. Desta forma, as indagações levantadas por Spalding \& Reeder (1972), Wisniewski \& Wilson (1992) e Thompson (1994) sobre as inconveniências da quimioterapia podem ser suplantadas pelo uso da termoterapia.

$\mathrm{O}$ uso de propiconazole associado à temperatura ambiente, $18^{\circ} \mathrm{C}$ e $13^{\circ} \mathrm{C}$ prolongou o período de conservação de bananas livres de doenças para 15, 24 (Figura 2) e 45 dias (dados não apresentados), respectivamente. No Brasil,

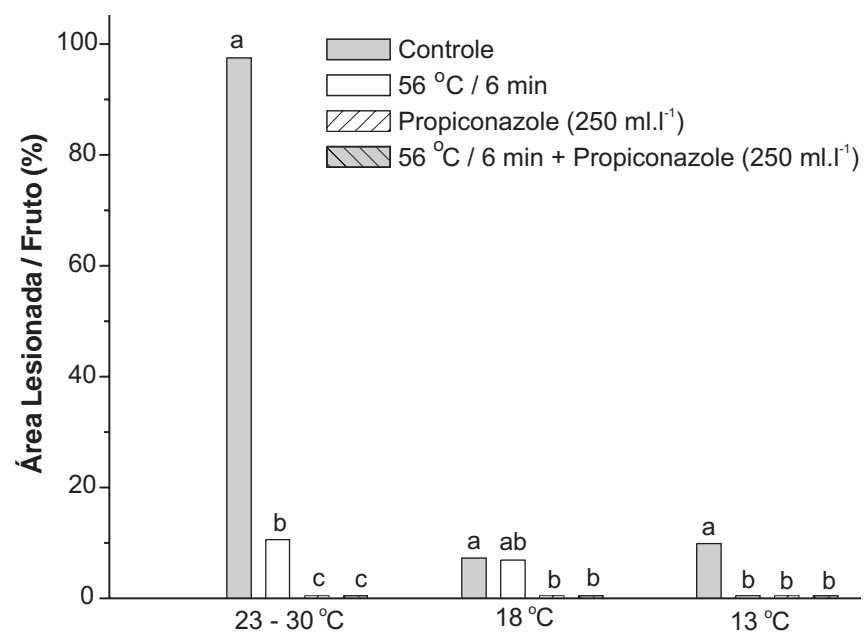

FIG. 1 - Efeito combinado da temperatura de armazenamento, termoterapia e quimioterapia sobre a percentagem de área lesionada por fruto de banana (Musa spp.) 'Prata-Anã' (AAB), 15 dias após os tratamentos. esse fungicida é recomendado na dosagem de $100 \mathrm{ml} . \mathrm{l}^{-1}$, como um dos mais eficientes no controle de doenças em póscolheita de bananas. Os resultados confirmam sua eficiência mesmo sob condições ambiente com elevada temperatura, porém na dosagem de $250 \mathrm{ml}^{-l^{-1}}$. Assim, tornase possível obter maior eficiência com dosagens menores, quando associadas à termoterapia $\left(56^{\circ} \mathrm{C}\right.$ durante $\left.6 \mathrm{~min}\right)$, e
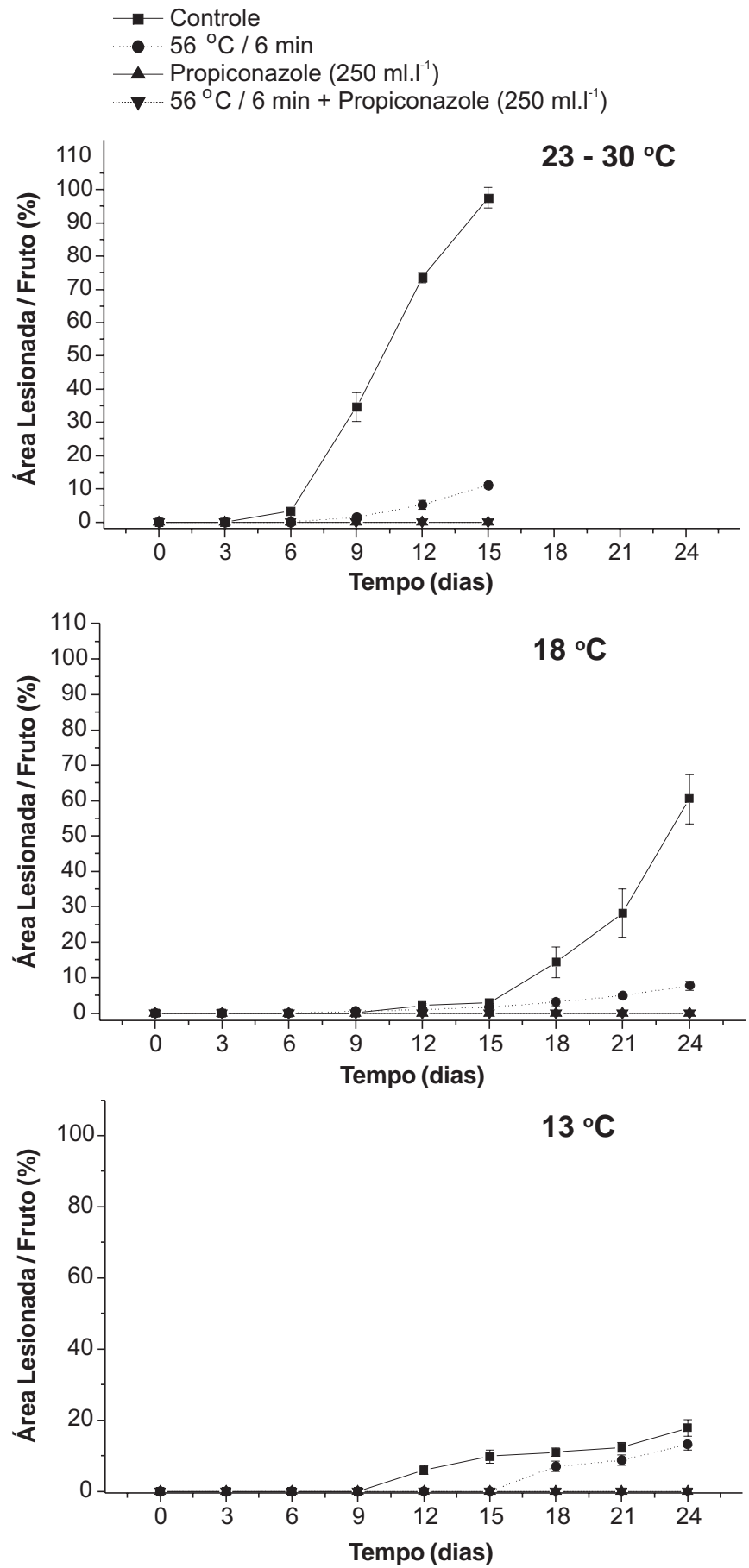

FIG. 2 - Evolução da área lesionada por fruto de banana (Musa spp.) 'Prata-Anã' (AAB) submetido à termoterapia e à quimioterapia armazenado sob diferentes temperaturas. 
principalmente com a temperatura de armazenamento de 13 ${ }^{\circ} \mathrm{C}$, mantendo níveis mais toleráveis de resíduos nos frutos.

O armazenamento dos frutos a $13{ }^{\circ} \mathrm{C}$ não retardou o início de manifestações de podridões, uma vez que os primeiros sintomas foram observados aos nove dias em frutos que não receberam tratamento adicional (Figura 2). Porém, nessa temperatura houve redução da percentagem de área lesionada por fruto (Figura 1) e redução da perda de peso (Figura 3).

Uma vez que, o armazenamento refrigerado a $13{ }^{\circ} \mathrm{C}$ não foi suficiente para conter as podridões, tratamentos adicionais são necessários, quer seja térmico, químico ou combinados, para garantir o completo controle das podridões. Segundo Lichtemberg et al. (2001) bananas do subgrupo Prata podem ser expostas a temperaturas inferiores a $12{ }^{\circ} \mathrm{C}$ no armazenamento, sem ocorrência de injúria por frio (chilling), o que poderia facilitar no transporte a longas distâncias e reduzir a incidência de podridões.

A percentagem de perda de peso foi influenciada significativamente tanto pela temperatura de armazenamento como pelos tratamentos utilizados, uma vez que ao final das avaliações os frutos do tratamento controle (não tratados) apresentaram percentagem média de perda de peso de 25 , 10 e 3\%, da termoterapia de 24, 11 e 5\%, do tratamento químico de 20, 10 e 3\% e do térmico+químico de 19, 10 e $5 \%$, quando associados à temperatura ambiente, $18^{\circ} \mathrm{C}$ e $13^{\circ} \mathrm{C}$, respectivamente (Figura 3 e 4).

Diferenças na perda de peso foram observadas apenas entre tratamentos mantidos sob temperatura ambiente, onde a termoterapia assemelhou-se ao tratamento controle (não tratado) e o tratamento químico ao térmico+químico. Contudo, redução gradual de perda de peso foi observada entre as temperaturas de armazenamento, resultando na redução máxima de $22 \%$ para o tratamento controle mantido

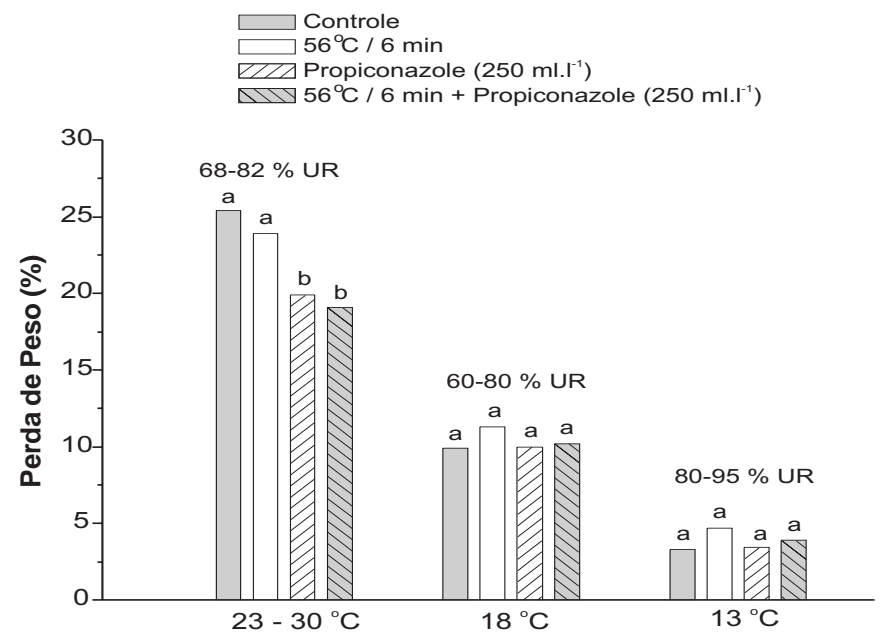

FIG. 3 - Efeito combinado da temperatura de armazenamento, termoterapia e quimioterapia sobre a percentagem de perda de peso de fruto de banana (Musa spp.) 'Prata-Anã' (AAB), 15 dias após os tratamentos. à temperatura ambiente e $13^{\circ} \mathrm{C}$, após 15 dias (Figura 3 e 4).

A coloração externa da casca dos frutos, medida pelo índice de cor da casca, foi influenciada isoladamente pela temperatura de armazenamento (Figura 5) e pelos tratamentos utilizados (Figura 6), não havendo, portanto, interação signifi-
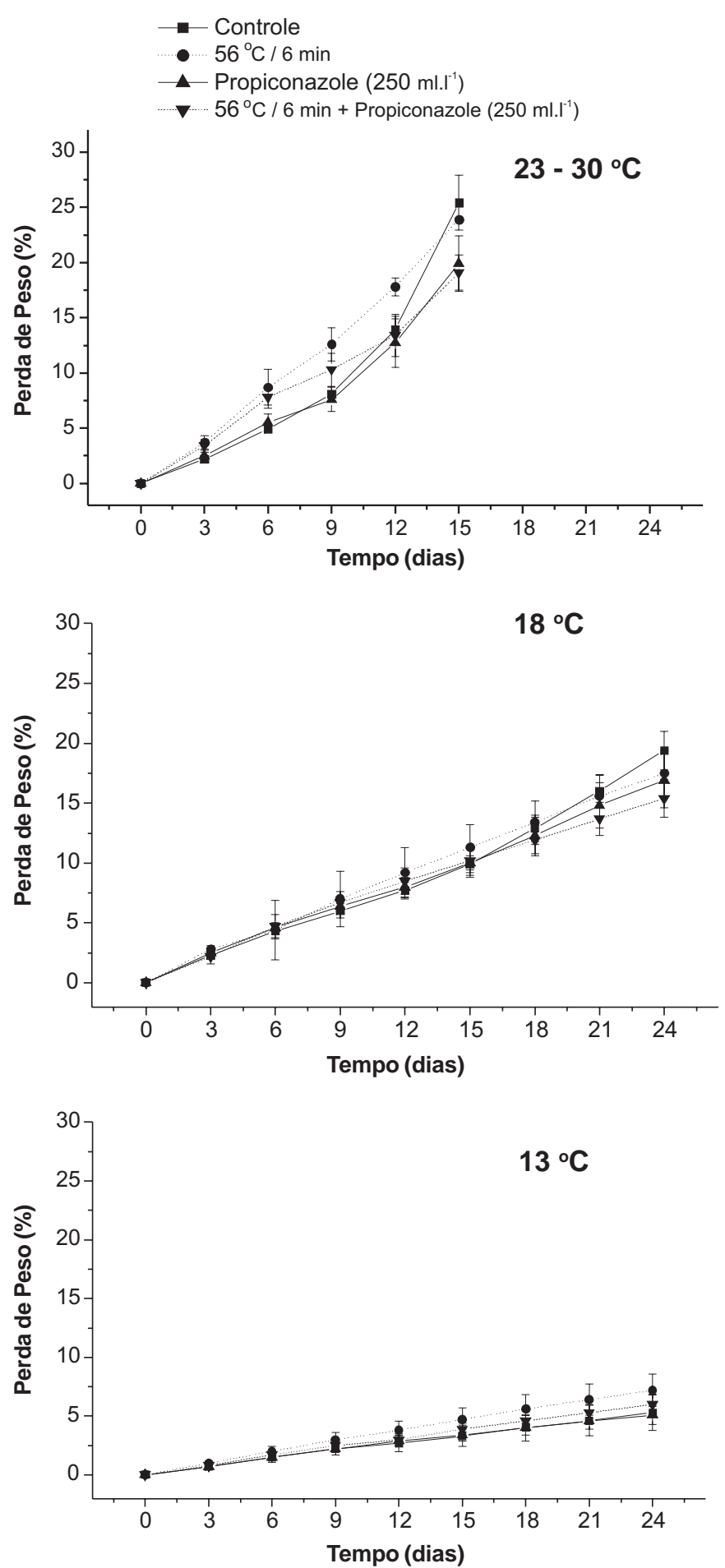

FIG. 4 - Evolução da perda de peso de frutos de banana (Musa sp.) 'Prata-Anã' (AAB) submetidos à termoterapia e à quimioterapia e armazenados sob diferentes temperaturas. 
cativa entre esses fatores. Frutos mantidos a $18{ }^{\circ} \mathrm{C}$ e $13{ }^{\circ} \mathrm{C}$ atingiram índice médio de cor 4,3 (cor intermediária) e 1 (verde), respectivamente, enquanto, frutos mantidos à temperatura ambiente atingiram 6,3 (amarelo), ocorrendo retardamento do amadurecimento aos 15 dias (Figura 5). Da mesma forma, a termoterapia e a quimioterapia apresentaram efeito semelhante sobre o amadurecimento dos frutos, mantendo índices médios de cor 4 e 3,5 respectivamente, diferindo do tratamento controle, que atingiu a índice médio de cor 4,5 aos 15 dias (Figura 6). Bananas 'Prata de Santa Catarina' e 'Dwarf Cavendish' também apresentaram atraso na evolução da cor da casca quando submetidas a termoterapia com temperaturas inferiores a $50^{\circ} \mathrm{C}$ (Dominguez et al., 1998).

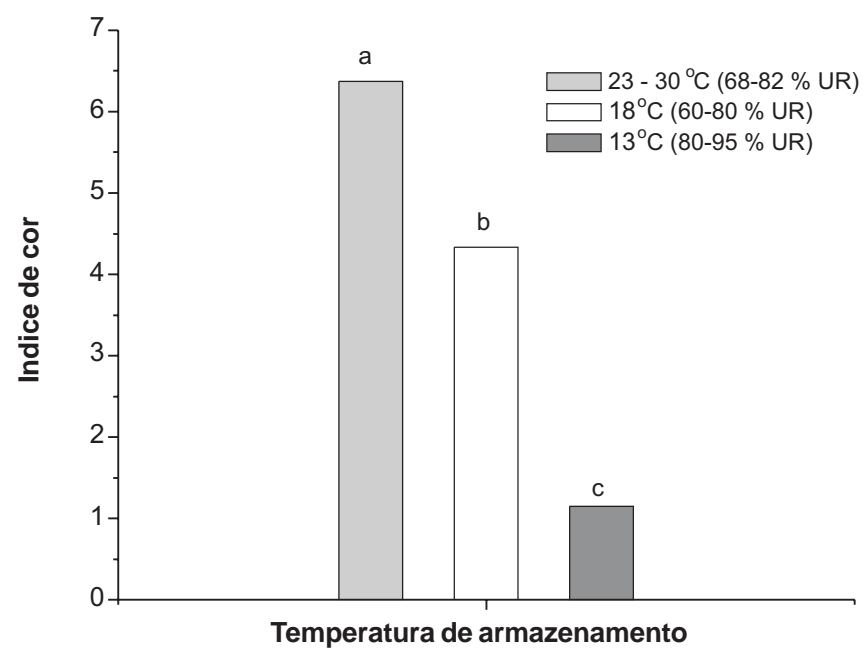

FIG. 5 - Efeito isolado da temperatura de armazenamento sobre o índice de cor da casca de frutos de banana (Musa sp.) 'Prata-Anã' (AAB), 15 dias após os tratamentos.

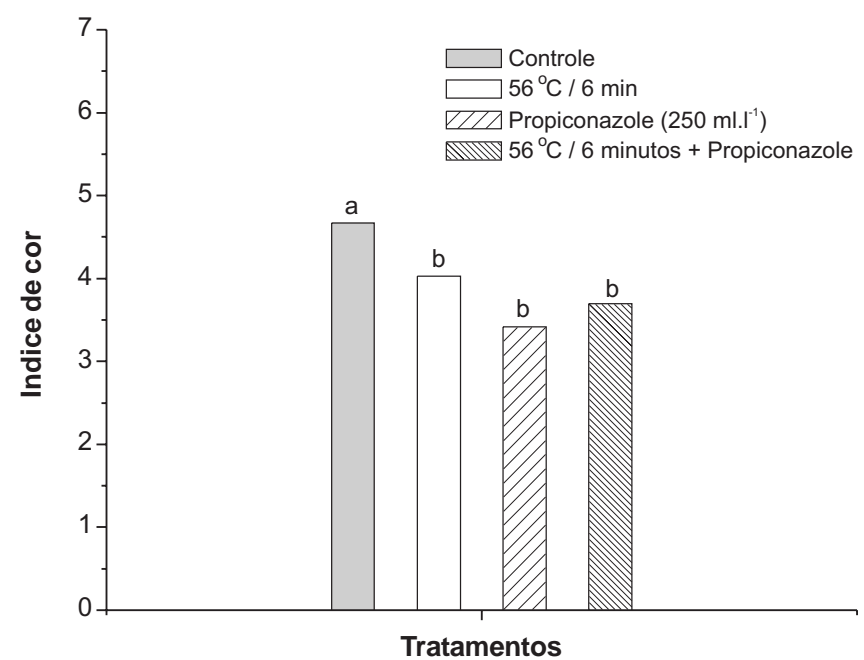

FIG. 6 - Efeito isolado da quimioterapia sobre o índice de cor da casca de frutos de banana (Musa sp.) 'Prata-Anã' (AAB), 15 dias após os tratamentos.
Jones \& Slaubaugh (1994) também observaram atraso na evolução da cor em frutos de banana tratados com fungicida procloraz.

O período de conservação de bananas foi estendido até 18,24 e 45 dias, à temperatura ambiente, $18^{\circ} \mathrm{C}$ e $13^{\circ} \mathrm{C}$, respectivamente. Esses resultados atendem ao desafio citado por Alves et al. (1995), uma vez que a ação de microorganismos e a perda de peso foram controladas pela interação da termoterapia e/ou quimioterapia com a temperatura de armazenamento e o retardamento do amadurecimento controlado pela temperatura de armazenamento em pós-colheita.

Assim, o manejo da temperatura de armazenamento, associado aos métodos de controle de podridão, permitiu evitar a manifestação de podridões, reduzir a perda de peso, manter a coloração externa em índices desejáveis e garantir a qualidade dos frutos de banana 'Prata-Anã', provenientes de Jaíba, MG.

\section{REFERÊNCIAS BIBLIOGRÁFICAS}

ALVES, E.J., DANTAS, J.L.L. \& SOARES FILHO, W.S. Banana para exportação: aspectos técnicos da produção (Série Publicações Técnicas FRUPEX, 18). Brasília:EMBRAPA. 1995.

COUEY, M.H. Heat treatment for control post-harvest disease and inset pest of fruits. HortScience 24:98-202. 1989.

DADZIE, B.K. \& ORCHARD, J.E. Post-harvest criteria and methods for routine screening of banana and plantain hybrids. Proceedings, International Network for Improvement of Banana and Plantain. Montpellier, France. 1996.

DOMÍNGUEZ, A.M., LÓPEZ CABRERA, J.J. \& GARCÍA, M. P. Effects of hot water treatments on postharvest quality and ethylene synthesis of bananas. Acta Horticulturae 490:529-535. 1998.

DURIGAN, J.F. Armazenamento de mamões (Carica papaya L.) 'Improved Sunrise Solo Line 72/12', com uso de hidrotermia, fungicida, cera, filmes plásticos e $\mathrm{KMnO}_{4}$, associados a diferentes temperaturas de refrigeração. (Tese de Livre Docência). Jaboticabal:UNESP. 1994.

HEWAGE, S.K., WAINWRIGHT, H., SWINBURNE, T.N. \& WIJERATHNAN, R.S.W. Modified atmosphere storage of bananas at chilling temperatures. Proceedings, Postharvest handling of tropical fruits. Australia. 1994. pp.419-421.

HOCHAUS, K. Transporte frigorífico por mar, experiências e possibilidades. International Fruit World 52:235-241. 1994.

JACOBI, K., COATES, L. \& WONG, L. Heat desinfestation of mangoes: effect on fruit quality and disease control. Proceedings, Postharvest handling of tropical fruits, Australia. 1994. pp.280287.

JANKOVIC, M. The influence of ripening condictions on banana quality. Recuil des Travaux de Recherches de la Facultè Agrinomique 32-33:130-134. 1988.

JOHNSON, G.I. \& SANGCHOTE, S. Control post-harvest disease of tropical fruits:challanges for the 21st century. Proceedings, Postharvest handling of tropical fruits, Australia. 1994. pp.140161.

JONES, D.R. \& SLAUBAUGH, W.R. Banana disease caused by 
fungi:antracnose and fungal scald. In: Ploetz, C., Zentmyer, W.T., Nishigima, K.G. \& Rohrback, H.D. (Eds.). Compendium of tropical fruit diseases. St. Paul MN. APS Press. 1994. pp.4-5.

KADER, A.A. Modified and controlled atmosphere storage of tropical fruits. Proceedings, Postharvest handling of tropical fruits. Australia. 1994. pp.239-249.

LABAVITCH, J.M. Fruit ripening and defence against pathogens loss of resistence or gain of susceptibility. Proceedings, Disease resistance in fruit. Australia. 1998. pp.53-59.

LICHTEMBERG, L.H., MALBURG, J.L. \& HINZ, R.H. Suscetibilidade varietal de frutos de bananeira ao frio. Revista Brasileira de Fruticultura 23:568-572. 2001.

LIU, X., GUO. G., HUANG, S., LIU, X. J., HUANG, S. M. \& GUO, G. The research and utilization of postharvest heat treatment for fruit storage. South China Fruits 26:46-48. 1997.

SMITH, W.L. \& ANDERSON, R.E. Decay control of peaches and nectarines during and after controlled atmosphere and air storage. Journal of the American Society of Horticulture Science 100:8486. 1975.

SPALDING, D.H. \& REEDER, W.F. Postharvest disorders of mango as affected by fungicides and heat treatments. Plant Disease Reporter 56:751-753. 1972.

THOMPSON, AK. Preharvest fungicidal spray for postharvest disease control. Proceedings, Postharvest handling of tropical fruits. Australia 1994. pp.169-171.

WISNIEWSKI, M.E. \& WILSON, C.L. Biological control of postharvest disease of fruits and vegetables: Recent Advances. HortScience 27:94-98. 1992. 